\title{
Estimation of Fan Beam and Parallel Beam Parameters in a Wire Mesh Design
}

\author{
Abdollah Khorshidi ${ }^{1}$, Mansour Ashoor ${ }^{2}$, S. Hamed Hosseini ${ }^{1}$, and Azimeh Rajaee ${ }^{1}$ \\ ${ }^{1}$ Department of Physics, Science and Research Branch, Islamic Azad University, Tehran, Iran; and ${ }^{2}$ Nuclear Science and Technology \\ Research Institute, Atomic Energy Organization of Iran, Tehran, Iran
}

\begin{abstract}
Special collimators used in imaging systems play an important part in obtaining qualified images to improve diagnosis in medicine. Methods: The primary aim of this study was to compare resolution between fan beam and parallel beam collimators using Monte Carlo simulation in the shape of cubic holes. Also, parameters such as geometric efficiency, geometric resolution, scatter, penetration, and full width at half maximum were studied to compare their special characteristics. Results: The simulation results demonstrated that the geometry efficiency of a fan beam collimator increased as the angle of the slant hole increased, and the geometric resolution decreased as the angle of the slant hole increased, at a distinct distance from a monoenergetic source of $\gamma$-rays. In contrast, at a distinct angle, geometric resolution increased as the distance between the source and the collimator surface increased. For both collimators, scatter and penetration decreased as the distance increased. These results were in agreement with ADAC company data. Finally, fan beam collimators were found to have better resolution than parallel beam collimators with a cubic hole shape in a wire mesh design. Conclusion: Estimation of the fan beam by parallel beam parameters as cubic holes can be suitable in collimator design to improve resolution and efficiency.
\end{abstract}

Key Words: fan beam; cube; FWHM; MCNP; phantom

J Nucl Med Technol 2012; 40:37-43

DOI: 10.2967/jnmt.111.089904

\section{C} ollimation of low-energy photons, allowing detection of only those photons propagating in the appropriate direction, plays a key role in obtaining a suitable map in SPECT imaging. Some collimators, such as the parallel beam and fan beam types, are widely used in different applications and for organs centered within the field of view. Therefore, it is important to study collimator geometric properties to improve diagnosis in medicine. Fan beam collimators are a special type of converging collimator with the holes fo-

\footnotetext{
Received Mar. 3, 2011; revision accepted Oct. 27, 2011.

For correspondence or reprints contact: Abdollah Khorshidi, Department of Physics, Science and Research Branch, Islamic Azad University, P.O. Box 14155/4933, Tehran, Iran.

E-mail: abkhorshidi@yahoo.com

Published online Jan. 11, 2012.

COPYRIGHT @ 2012 by the Society of Nuclear Medicine, Inc.
}

cusing toward a focal line parallel to the axis of rotation of the camera ( 1 ).

For optimization of collimator design, photon interactions in $\gamma$-camera collimators have been simulated by the Monte Carlo N-particle code (MCNP5) (2-4). In this code, source geometry, collimators, and detectors need to be defined in the input file-a cumbersome process $(5,6)$. Recently, researchers have clinically compared parallel beam and fan beam collimators and simulated the parallel beam collimator by this code (7-22). In this study, fan beam parameters were deliberated by this code.

To improve resolution and efficiency, it is helpful to model collimators of various hole shapes, edge effects, septal materials, and geometric configurations. The aim of this study was to investigate resolution between fan beam and parallel beam collimators using MCNP5 simulation in the shape of cubic holes. Also, distinctive parameters such as geometric efficiency, scatter, penetration, and full width at half maximum (FWHM) have been compared, with assessment of the fan beam and parallel beam collimator responses.

\section{MATERIALS AND METHODS}

\section{Computation of Cubic Hole Angles}

In collimator design, static and dynamic parameters are considered. Static parameters include material and geometry, such as the shape and size of the holes and the degree of the angle. Dynamic parameters include the position of the source and the location of the collimator. In this study, the geometric characteristic of low-energy parallel beam and fan beam collimators used at ADAC were applied, as indicated in Table 1 (23). In view of research by Gunter et al. showing that parallel beam parameters can be chosen locally for fan beam collimators, we estimated the fan beam geometric dimensions by parallel beam collimator parameters (24).

The MCNP5 code and Matlab software (MathWorks, Inc.) were used to simulate collimators with cubic holes as shown in Figure 1. On the central $z$-axis, both the fan beam collimator and the parallel beam collimator have the same hole length. On the $x$-axis, as the offset distance changes, the hole length of the fan beam collimator increases because of the various angles and the hole size. 
TABLE 1

Geometric Characteristics of Parallel Beam and Fan Beam Collimators

\begin{tabular}{ll}
\hline \multicolumn{1}{c}{ Characteristic } & Value \\
\hline Thickness $(\mathrm{cm})$ & 3.28 \\
Hole size $(\mathrm{cm})$ & 0.140 \\
Septal width $(\mathrm{cm})$ & 0.0152 \\
Ratio of hole area to septal area & 0.7682 \\
\hline
\end{tabular}

According to ADAC company data, the focal length of the fan beam collimator is $50 \mathrm{~cm}$ and the slant hole cross section is variable (23). A simulated collimator was defined by a cubic region because the unit cell, when replicated, would produce the desired hole pattern in parallel beam mode, as indicated in Figure 1A. In fan beam mode, each hole must be considered, with different angles, hole sizes, and hole lengths and constant septa in a similar volume. The cross section of the holes is of a parallelepiped shape, and the hole angles on the fan beam collimator can be computed given the geometric parameters. As the angle increases, the cross section varies according to a row of holes focused on a point. Also, there is another row of holes centered on another focal point in the same direction at an equal distance from the collimator surface. The focal line is formed by the focal points. Figure 2 represents this objective with dissimilar diameters for 2 holes beside each other and can be defined as:

$$
\frac{r}{f-x}=\frac{\frac{s}{x}}{\sqrt{1+\frac{s^{2}}{x^{2}}}},
$$

where $r$ is the distance between the wall and center of a hole, $s$ is the septal thickness, $f$ is the focal line distance, and $x$ is the variable parameter in any angle of a slant hole.

\section{Monte Carlo Simulations}

Each camera system has a constant intrinsic resolution, depending on detector thickness, area, and substance density. This study used a NaI detector with a thickness of $0.95 \mathrm{~cm}$ in a $40 \times 54 \mathrm{~cm}$ area, and a scintillation camera with an intrinsic resolution of $0.39 \mathrm{~cm}$ was simulated by MCNP5. Several parameters were evaluated, such as FWHM, geometric resolution, and geometric efficiency for 89,600 slant holes and 89,600 cross sections.
FIGURE 1. (A) parallel beam collimator and $(B)$ fan beam collimator with cubic holes.

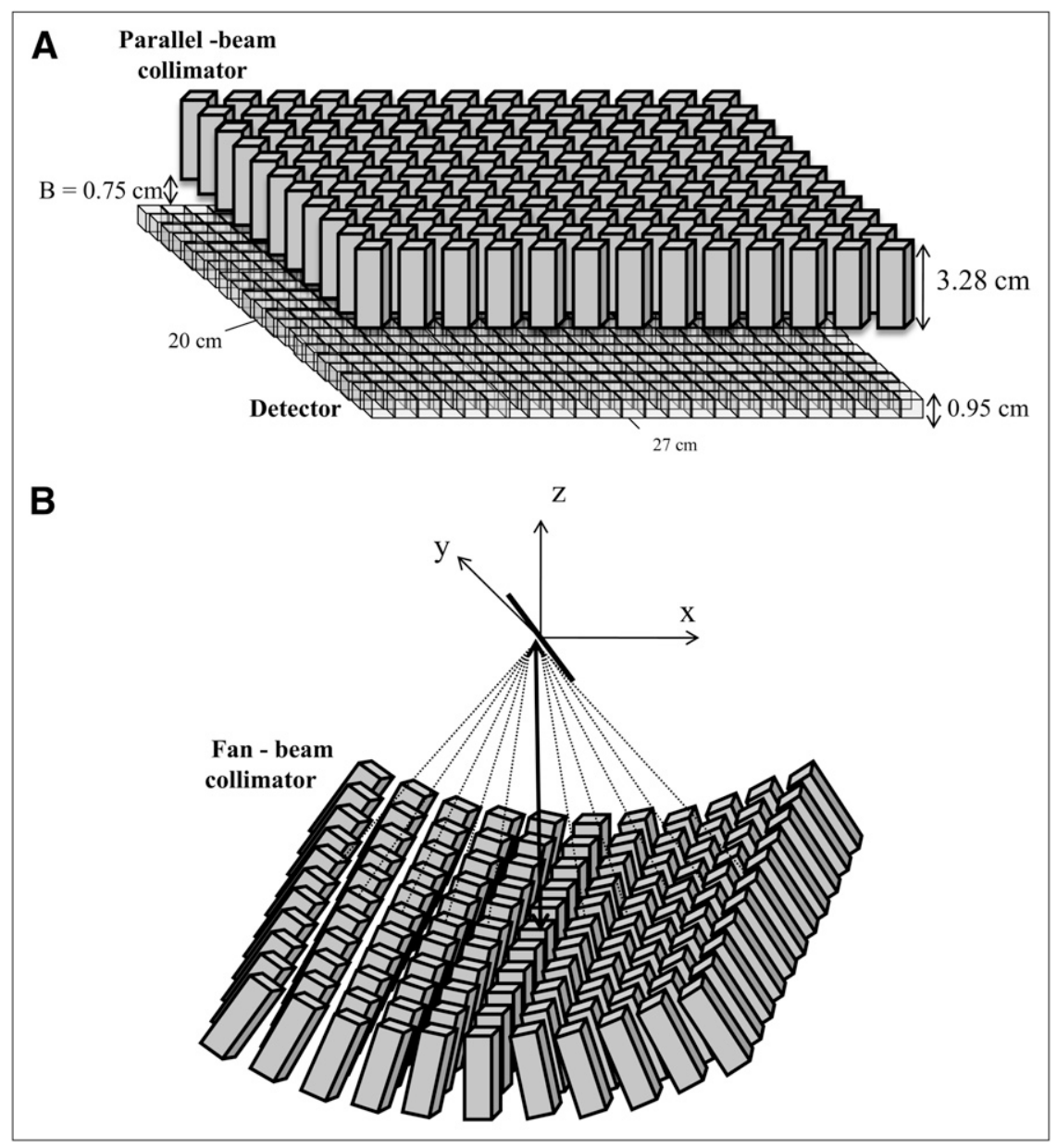




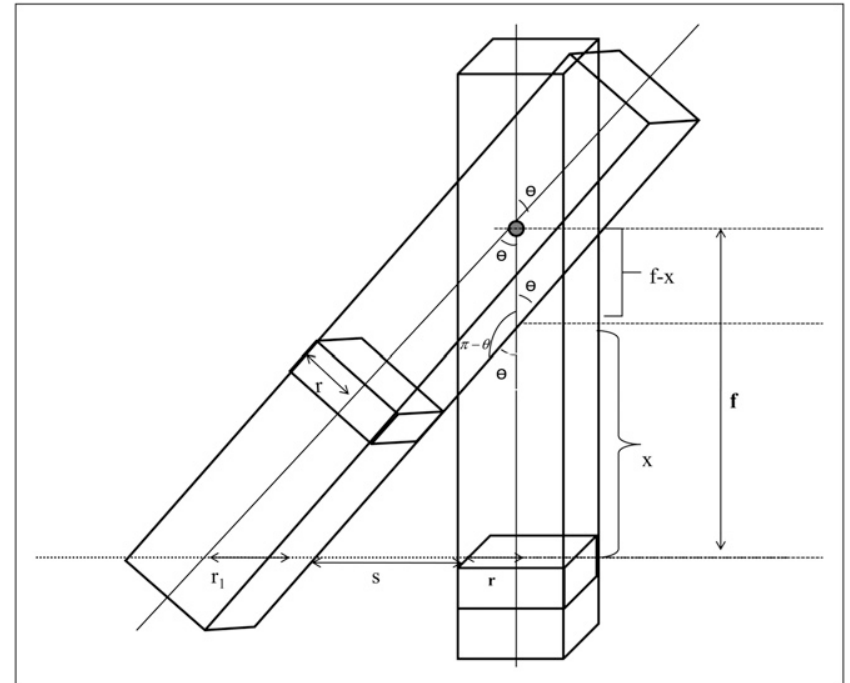

FIGURE 2. Calculation of angles in fan beam collimator, in which slanted cubic hole has different cross sections.

In fan beam collimator simulation, angles were calculated for 89,600 slant holes in a $40 \times 54 \times 3.28 \mathrm{~cm}$ region with a septal thickness of $0.0152 \mathrm{~cm}$ and various cross sections, in which the holes focused to a line. Metz et al. (22) derived a closed algebraic expression to determine the

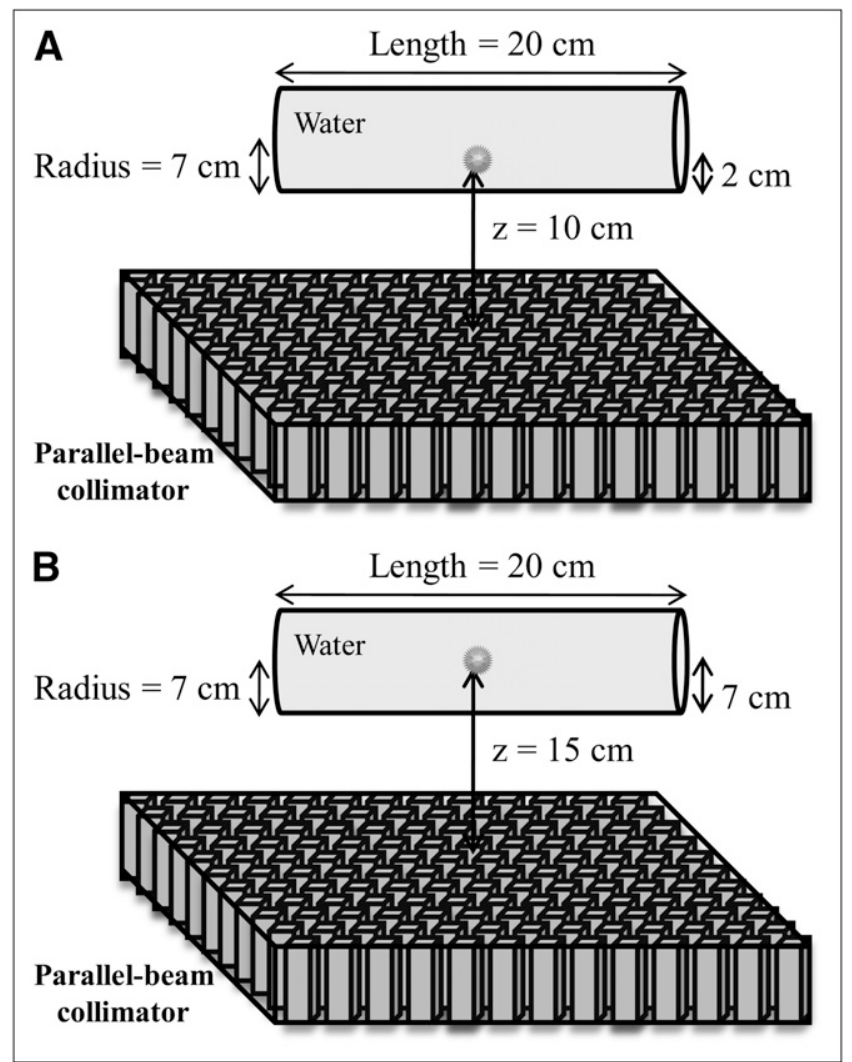

FIGURE 3. Point source in scatter medium in front of collimator at $z=10 \mathrm{~cm}(\mathrm{~A})$ and $z=15 \mathrm{~cm} \mathrm{(B).}$
TABLE 2

Simulated Parallel Beam Effective and Total Resolutions at Different $z$ Values

\begin{tabular}{lcc}
\hline & \multicolumn{2}{c}{$\begin{array}{c}\text { Source-collimator surface } \\
\text { distance }(\mathrm{cm})\end{array}$} \\
\cline { 2 - 3 } Resolution $(\mathrm{cm})$ & $z=10$ & $z=15$ \\
\hline Effective & 0.6119 & 0.8314 \\
Total & 0.7256 & 0.9183
\end{tabular}

Effective hole length $=3.189 \mathrm{~cm} ; \mu=22 \mathrm{~cm}^{-1}$ for lead at 140 $\mathrm{keV}$ and $\mathrm{B}=0.75 \mathrm{~cm}$.

geometric component of the parallel beam collimators and found that if penetration is negligible, the spatially geometric component is effectively independent of hole shape for a given collimator thickness and a given ratio of hole area to septal area. Table 1 indicates the geometric properties of the collimators used for the simulation.

The point source, technetium $(140 \mathrm{keV})$, was placed in air and medium (water phantom) to obtain adequate scatter, penetration, and point spread. This source was located at $z=10$ and $15 \mathrm{~cm}$ in front of the collimator surface, and energy spectra with a $20 \%$ photopeak window were obtained (25). In each case, 500 million photons were detected by the $\mathrm{NaI}$ detector.

The phantom was considered a cylinder with a length of $20 \mathrm{~cm}$ and a radius of $7 \mathrm{~cm}$ to study source depth in tissue. The depths were assumed to be $2 \mathrm{~cm}(z=10 \mathrm{~cm})$ and $7 \mathrm{~cm}$ $(z=15 \mathrm{~cm})$, as shown in Figure 3. Meanwhile, for calculation of scatter and penetration, simulations were performed with and without interactions in collimators of the same sensitivity.

TABLE 3

Calculated Fan Beam Geometric Efficiency $\left(\times 10^{-5}\right)$ at Various $z$ Values and Angles

\begin{tabular}{ccc}
\hline & \multicolumn{2}{c}{$\begin{array}{c}\text { Source-collimator surface } \\
\text { distance }(\mathrm{cm})\end{array}$} \\
\cline { 2 - 3 } Angle (degree) & $z=10$ & $z=15$ \\
\hline 1.6216 & 12.0024 & 15.6770 \\
3.2419 & 11.9449 & 15.6010 \\
4.8595 & 11.8497 & 15.4770 \\
6.4733 & 11.7180 & 15.3050 \\
8.0818 & 11.5512 & 15.0870 \\
\hline .6839 & 11.3510 & 14.8260 \\
11.2783 & 11.1196 & 14.5236 \\
12.8638 & 10.8595 & 14.1838 \\
14.4393 & 10.5732 & 13.8099 \\
\hline 16.0035 & 10.2636 & 13.4055 \\
17.5555 & 9.9337 & 12.9746 \\
\hline 19.0942 & 9.5864 & 12.5211 \\
20.6186 & 9.2250 & 12.0490 \\
22.1277 & 8.8523 & 11.5623 \\
23.6206 & 8.4715 & 11.0648 \\
\hline
\end{tabular}




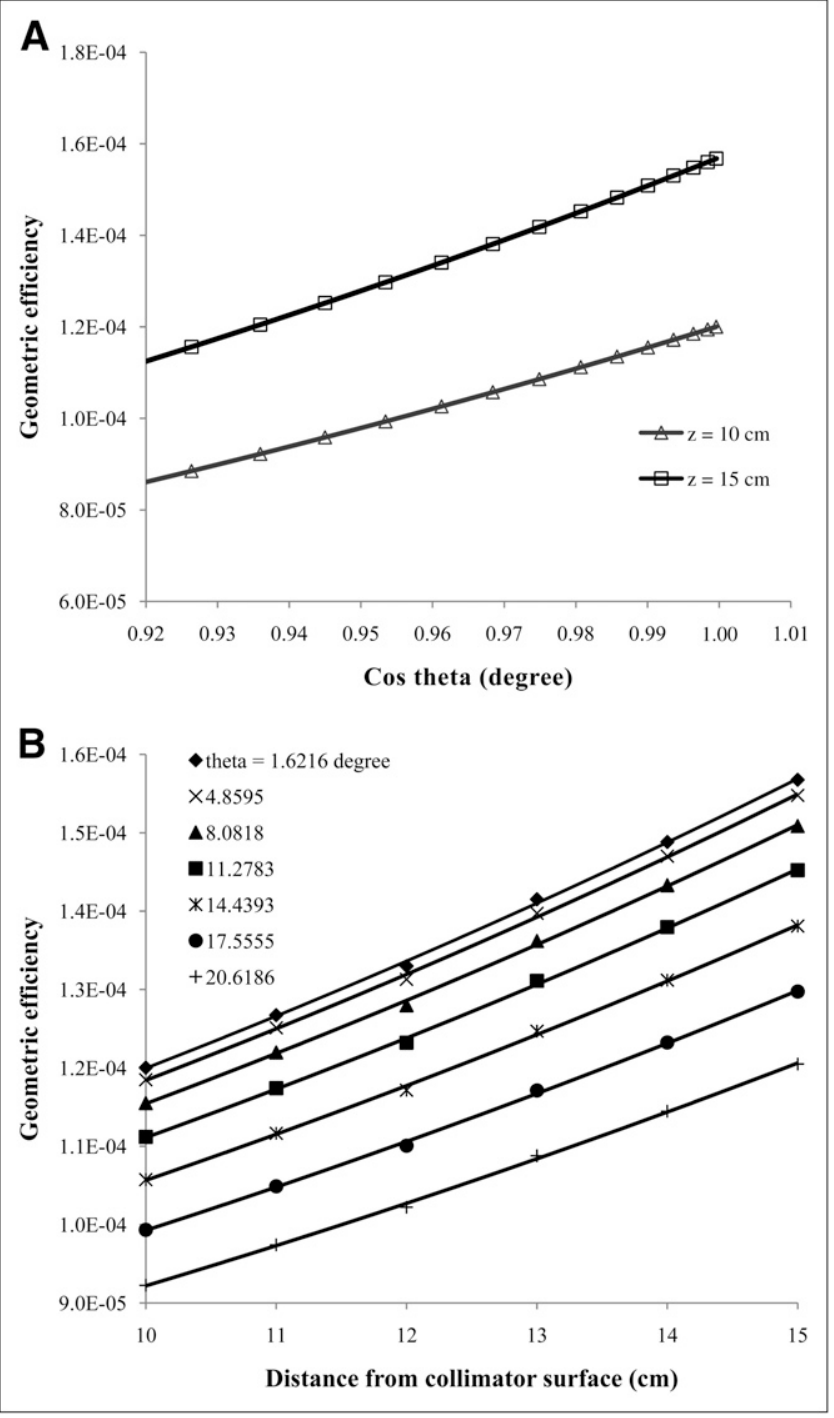

FIGURE 4. (A) Simulated fan beam geometric efficiency lessens when angle of slant hole is increased. (B) Simulated fan beam geometric efficiency increases when point source distance from collimator surface is increased for different $\theta$ s.

\section{Calculation and Assessment of Collimator Parameters}

The ratio of hole area $\left(A_{\text {open }}\right)$ to septal area $\left(A_{\text {unit }}\right)$ can be defined in the geometric efficiency of the parallel beam collimator $\left(G_{P B}\right)$ as follows:

$$
\begin{aligned}
& G_{P B}=\left(\frac{A_{\text {open }}}{4 \pi l_{e}^{2}}\right)\left(\frac{A_{\text {open }}}{A_{\text {unit }}}\right), \\
& l_{e}=l-2 \mu^{-1}
\end{aligned}
$$

where $l_{e}$ is the effective hole length of the collimator and $\mu$ is the linear attenuation coefficient of the septal material (lead) at $140 \mathrm{keV}(26,27,1)$. The effective geometric spatial resolution $\left(R_{c}\right)$ is described as the FWHM of the intensity distribution obtained from a point source placed at a distance from the collimator face (2), given by:
TABLE 4

Calculated Fan Beam Effective Resolution at Various Distances from Collimator Surface

\begin{tabular}{ccc}
\hline & \multicolumn{2}{c}{$\begin{array}{c}\text { Source-collimator surface } \\
\text { distance }(\mathrm{cm})\end{array}$} \\
\cline { 2 - 3 } Angle (degree) & $z=10$ & $z=15$ \\
\hline 1.6216 & 0.4274 & 0.5036 \\
3.2419 & 0.4279 & 0.5042 \\
4.8595 & 0.4287 & 0.5052 \\
\hline 6.4733 & 0.4299 & 0.5066 \\
\hline 8.0818 & 0.4315 & 0.5084 \\
\hline 9.6839 & 0.4334 & 0.5106 \\
11.2783 & 0.4356 & 0.5133 \\
\hline 12.8638 & 0.4382 & 0.5163 \\
14.4393 & 0.4411 & 0.5198 \\
16.0035 & 0.4444 & 0.5236 \\
17.5555 & 0.4480 & 0.5279 \\
\hline 19.0942 & 0.4521 & 0.5326 \\
\hline 20.6186 & 0.4564 & 0.5378 \\
\hline 22.1277 & 0.4612 & 0.5434 \\
\hline 23.6206 & 0.4663 & 0.5494 \\
\hline
\end{tabular}

$$
R_{c}=\frac{a\left(l_{e}+z+b\right)}{l_{e}}
$$

Eq. 3

where $a$ is hole size, $b$ is distance between the crystal and the collimator, and $z$ is distance between the source and the collimator surface. Also, total resolution can be defined as:

$$
R_{t}=\sqrt{R_{c}^{2}+R_{i}^{2}}
$$

Eq. 4

The geometric efficiency of the fan beam collimator $\left(G_{F B}\right)$ is expressed as follows (3):

TABLE 5

Calculated Fan Beam Total Resolution at Various Distances from Collimator Surface

\begin{tabular}{ccc}
\hline & \multicolumn{2}{c}{$\begin{array}{c}\text { Source-collimator surface } \\
\text { distance }(\mathrm{cm})\end{array}$} \\
\cline { 2 - 3 } Angle (degree) & $z=10$ & $z=15$ \\
\hline 1.6216 & 0.5152 & 0.5636 \\
3.2419 & 0.5156 & 0.5641 \\
4.8595 & 0.5163 & 0.5650 \\
6.4733 & 0.5173 & 0.5663 \\
8.0818 & 0.5186 & 0.5679 \\
\hline .6839 & 0.5202 & 0.5699 \\
11.2783 & 0.5221 & 0.5722 \\
12.8638 & 0.5242 & 0.5750 \\
14.4393 & 0.5267 & 0.5781 \\
16.0035 & 0.5294 & 0.5816 \\
17.5555 & 0.5325 & 0.5855 \\
19.0942 & 0.5359 & 0.5897 \\
20.6186 & 0.5395 & 0.5944 \\
22.1277 & 0.5436 & 0.5994 \\
23.6206 & 0.5479 & 0.6049 \\
\hline
\end{tabular}




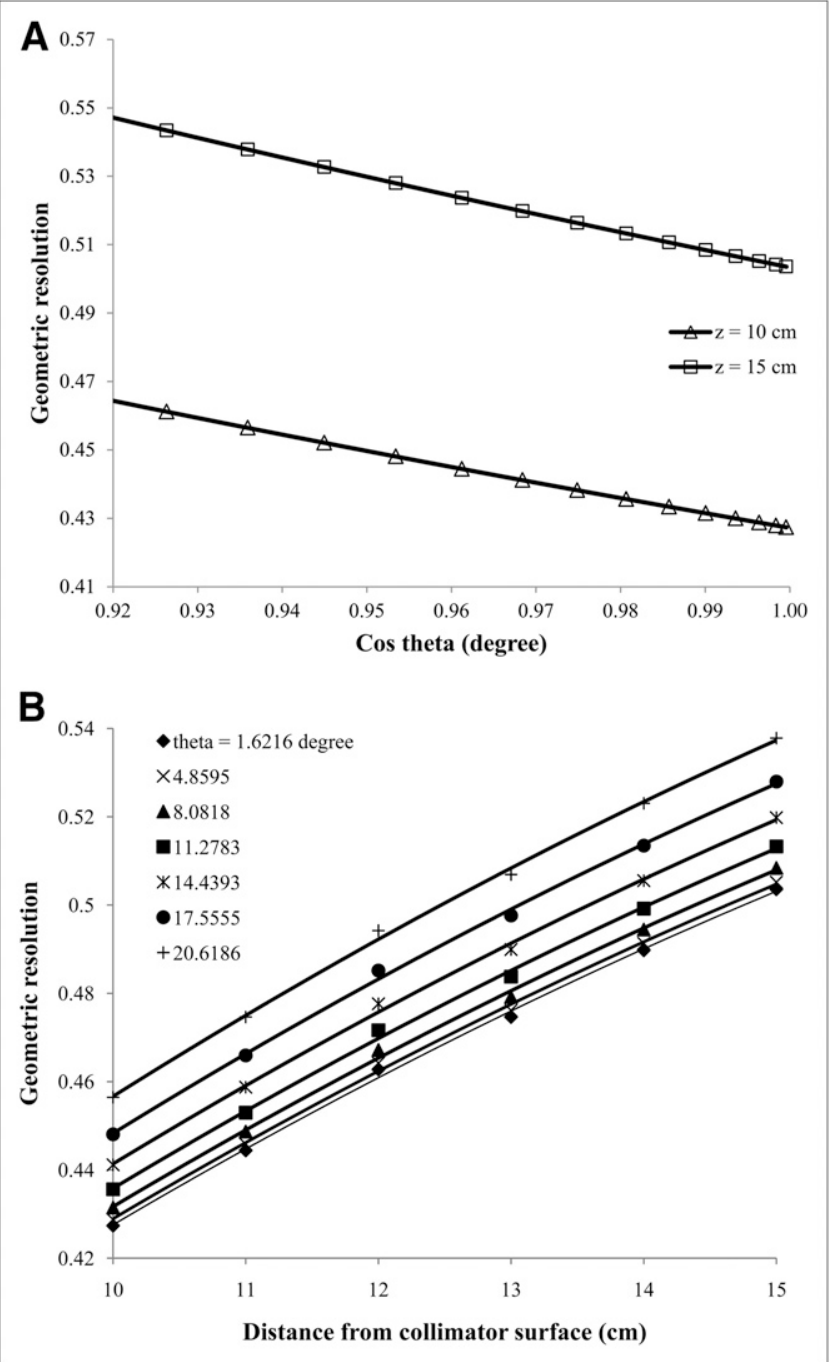

FIGURE 5. (A) Simulated fan beam geometric resolution decreases when angle of cubic hole is reduced for different $z$ values. (B) Simulated effective resolution increases when angle of holes is increased.

$$
\begin{aligned}
& G_{F B}=\left(\frac{A_{\text {open }}}{4 \pi l_{e}^{\prime 2}}\right) \times\left(\frac{A_{\text {open }}}{A_{\text {unit }}}\right) \times\left(\frac{f}{f-z}\right)^{2} 0 \leq z \leq f . \\
& l_{e}^{\prime}=\frac{l_{e}}{\cos \theta}
\end{aligned}
$$

In the fan beam collimator design, the $\cos \theta$ term takes into account the oblique holes, which are not on the central axis. Because of the magnification property of the fan beam collimator, the geometric spatial resolution is given by (23):

$$
R_{c}=\left[\frac{a\left(l_{e}+z+b\right)}{l_{e}} \times \frac{1}{\cos \theta} \times\left(1-\frac{\frac{l_{e}}{2}+b}{f-z}\right)\right] \times\left[\frac{f-z}{f+l_{e}+b}\right] . \quad \text { Eq. } 6
$$

The total geometric spatial resolution of the fan beam collimator is given by:

$$
R_{t}=\sqrt{R_{c}^{2}+\left(\frac{f-z}{f+l_{e}+b}\right)^{2} R_{i}^{2}}
$$

where intrinsic resolution is the intrinsic spatial resolution of the camera.

\section{RESULTS}

The effective and total resolutions increased when the distance between the source and the parallel beam collimator increased, as shown in Table 2. The effective and total resolutions reported by $\mathrm{ADAC}$ at a $10-\mathrm{cm}$ distance for the parallel beam collimator were 0.63 and $0.74 \mathrm{~cm}$, respectively. These values agree with our results, with errors of $3.2 \%$ and $2.7 \%$, respectively. Also, the geometric efficiency of the parallel beam collimator and effective hole length were calculated to be $7.69387 \mathrm{E}-05$ and $3.189 \mathrm{~cm}$, respectively.

The geometric efficiency of a fan beam collimator is related to angle and distance and, for a distinct angle, could be increased by increasing the distance, as indicated in Table 3. Geometric efficiency could also be decreased by increasing the slant hole angle, as shown in Figure 4.

The effective and total resolutions of a fan beam collimator with 89,600 holes, for all angles, were calculated to be 0.59 and $0.68 \mathrm{~cm}$, respectively, at a $10-\mathrm{cm}$ distance. The reported effective and total resolutions from ADAC under the same conditions were 0.60 and $0.71 \mathrm{~cm}$, respectively. At a distance of $15 \mathrm{~cm}$, the respective calculated values were 0.69 and $0.74 \mathrm{~cm}$, as shown in Tables 4 and 5

\begin{tabular}{|c|c|c|c|c|}
\hline \multirow[b]{3}{*}{ Collimator } & \multicolumn{4}{|c|}{ Source-collimator surface distance $(\mathrm{cm})$} \\
\hline & \multicolumn{2}{|c|}{$z=10$} & \multicolumn{2}{|c|}{$z=15$} \\
\hline & Air & Phantom $(2 \mathrm{~cm})$ & Air & Phantom $(7 \mathrm{~cm})$ \\
\hline System parallel beam & 0.80 & 0.87 & 0.95 & 1.22 \\
\hline Geometric parallel beam & 0.59 & & 0.86 & \\
\hline System fan beam & 0.79 & 0.81 & 0.91 & 1.16 \\
\hline Geometric fan beam & 0.58 & & 0.82 & \\
\hline
\end{tabular}

TABLE 6

Simulated FWHM (cm) for Parallel Beam and Fan Beam Collimators with Cubic Hole While Point Sources Are Located in Air and Water Phantoms 


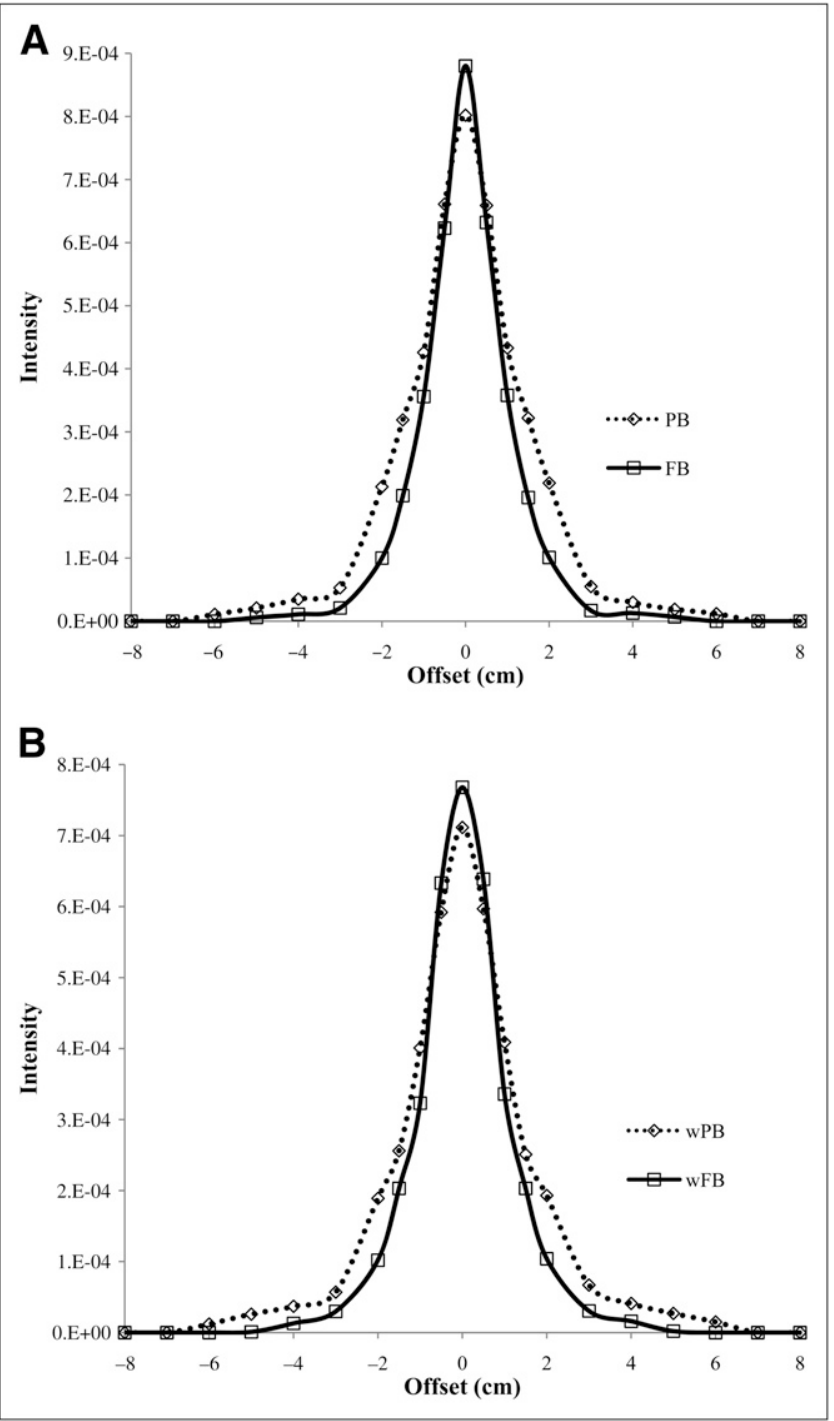

FIGURE 6. Simulated radial point spread functions for fan beam and parallel beam with cubic hole shape when point source is set at $z=10 \mathrm{~cm}$ in air phantom (A) and water phantom (B). FB = fan beam; $P B=$ parallel beam; $w=$ water.

and Figure 5. The values increased with an increase in the hole angle at a distinct distance, as well as with an increase in distance at a distinct angle.

For both collimators, the FWHM using cubic holes was investigated by radial point spread functions. FWHM increased as the distance increased, as shown in Table 6. With cubic holes, the FWHM of the fan beam collimator is less than that of the parallel beam collimator and results in a better resolution. Also, the radial point spread functions were simulated in a cubic shape at a $10-\mathrm{cm}$ distance in air by MCNP5. The fan beam collimator has better resolution than the parallel beam collimator, as shown in Figure 6A. The FWHM of collimators in the water phantom was increased because of Compton scattering, and the resolution was decreased, as indicated in Figure 6B.

Table 7 presents relative differences between the simulated and the reported resolutions at a $10-\mathrm{cm}$ distance from collimators including 89,600 holes: $6 \%$ and $3 \%$ for the parallel beam and fan beam collimators, respectively. Table 8 indicates the scatter and penetration values in air and water for a $20 \%$ photopeak window at various distances. For both collimators, scatter and penetration decreased as distance increased. At a distinct distance, scatter and penetration were found to be higher for the fan beam collimator than for the parallel beam collimator in both air and water because of the hole shape, geometric pattern, and edge effect.

\section{DISCUSSION}

This study investigated the resolution and geometric efficiency of parallel beam and fan beam collimators with cubic holes. The spatial resolution of a scintillation camera stems from a combination of the type of collimator and the intrinsic resolution characteristics of the crystal and electronic detection system. When the angle of the hole is changed, efficiency and resolution are altered, and vice versa. Thus, collimator parameters are designed with regard to the necessary trade-off between efficiency and resolution.

Cot et al. (28) indicated the square holes give rise to sensitivity higher than the hexagonal holes without considering the effective length and efficiency in actual extensive collimators. In this study, the simulations included all components plus photoelectric and electron $\mathrm{x}$-rays with higher accuracy $(<0.02)$.

To study the effects of interactions, the simulations included penetration, Compton and coherent scatter, photoelectric effects, and emission of characteristic lead x-rays. The programs also included interactions in a NaI scintillation crystal, and backscatter from a compartment representing detector components behind the crystal. The results of the simulation demonstrated that with an increase in distance there is a decrease in scatter and penetration. The fan beam had higher scatter and penetration, and the parallel beam had lower values, with a 1.04 ratio in air

TABLE 7

Comparison of Simulated FWHM (cm) with Values Reported by ADAC at $z=10 \mathrm{~cm}$ with 89,600 Holes

\begin{tabular}{lcccc}
\hline \multirow{2}{*}{ Resolution } & \multicolumn{2}{c}{ Parallel beam } & \multicolumn{2}{c}{ Fan beam } \\
\cline { 2 - 5 } \cline { 3 - 5 } & Simulated & Reported & Simulated & 0.60 \\
\hline Effective resolution & $0.59 \pm 0.02$ & 0.63 & $0.58 \pm 0.02$ & Reported \\
Total resolution & $0.80 \pm 0.02$ & 0.74 & $0.79 \pm 0.02$ & 0.71 \\
\hline
\end{tabular}


TABLE 8

Amount of Simulated Penetration and Scatter Percentage in Air and Water Phantoms Related to Distance from Collimator Surface

\begin{tabular}{cccccc}
\hline & \multicolumn{2}{c}{$z=10 \mathrm{~cm}$} & & \multicolumn{2}{c}{$z=15 \mathrm{~cm}$} \\
\cline { 2 - 3 } \cline { 5 - 6 } Collimator & Air & Phantom & & Air & Phantom \\
\hline Parallel beam & 3.07 & 3.25 & & 1.72 & 1.97 \\
Fan beam & 3.20 & 5.73 & & 2.15 & 3.21 \\
\hline
\end{tabular}

and 1.76 in phantom at $z=10 \mathrm{~cm}$. Scatter and penetration depend on the hole shape, geometric pattern, and edge effect. When scatter and penetration are not negligible, the point spread function will have a long tail, which will not affect FWHM but can significantly affect the contrast resolution of the collimator. Although system resolution was higher than geometric resolution, the fan beam, in comparison with the parallel beam with cubic holes, had the lowest system and geometric resolutions for a distinct distance.

\section{CONCLUSION}

This comparison demonstrated that simulated responses were close to the experimental data provided by ADAC. Compared with the parallel beam collimator, the fan beam collimator with a cubic hole shape resulted in a smaller full width at half maximum and better resolution. Modeling of collimators can be commercially beneficial to the manufacturing industry. In addition, modeling can be clinically relevant in allowing for the design of collimators with better qualities and features.

\section{ACKNOWLEDGMENT}

No potential conflict of interest relevant to this article was reported.

\section{REFERENCES}

1. Koole M, Asselerx YD, Vandenberghex S, et al. Modeling of the sensitivity of fan beam collimation in SPECT imaging. IEEE Eng Med Biol. 2001;3:75-78.

2. Vries DJD, Moore SC. Comparison of hexagonal-hole and square-hole collimation by Monte Carlo simulation. IEEE Trans Nucl Sci. 2001;3:52-56.

3. Gullberg GT, Tsui BM, Crawford CR, Edgerton ER. Estimation of geometrical parameters for fan beam tomography. Phys Med Biol. 1987;32:1581-1594.

4. Tusi BM, Simimons GH, eds. Collimator Design, Properties, and Characteristics in the Scintillation Camera. Reston, VA: Society of Nuclear Medicine; 1988:17-45.

5. Yancht JC, Dobrzenieckit AB, Ramanathant C, Behrmanl R. Physically realistic Monte Carlo simulation of source, collimator and tomographic data acquisition for emission computed tomography. Phys Med Biol. 1992;37:853-870.
6. Briesmeister JF. MCNP Data Manual. Los Alamos, NM: Los Alamos National Laboratory; 2001

7. Buvat I, Laffont S, Cloirec JL, Bourguet P, Paola RD. Importance of the choice of the collimator for the detection of small lesions in scintimammography: a phantom study. Phys Med Biol. 2001;46:1343-1355.

8. Celler A, Sitek A, Stoub E, Hawman P, Harrop R, Lyster D. Multiple line source array for SPECT transmission scans: simulation, phantom and patient studies. J Nucl Med. 1998;39:2183-2189.

9. Kim HJ, Karp JS, Mozley PD, et al. Simulating technetium-99m cerebral perfusion studies with a three-dimensional Hoffman brain phantom: collimator and filter selection in SPECT neuroimaging. Ann Nucl Med. 1996;10:153-160.

10. Gilland DR, Jaszczak RJ, Greer KL, Coleman RE. Transmission imaging for nonuniform attenuation correction using a three-headed SPECT camera. $J \mathrm{Nucl}$ Med. 1998;39:1105-1110.

11. Matsunari I, Boning G, Ziegler SI, et al. Effects of misalignment between transmission and emission scans on attenuation-corrected cardiac SPECT. J Nucl Med. 1998;39:411-416.

12. Tsui BM, Gullberg GT. The geometric transfer function for cone and fan beam collimators. Phys Med Biol. 1990;35:81-93.

13. Husák V, Perinova V. The design of collimators for radioisotope scanning. Phys Med Biol. 1969;14:233-244.

14. Eckholt M, Bergmann H. Angulation errors in parallel hole and fanbeam collimators: computer controlled quality control and acceptance testing procedure. J Nucl Med. 2000;41:548-555.

15. Beekman FJ, Kamphuis C, Hutton BF, Rijk PV. Half-fanbeam collimators combined with scanning point sources for simultaneous emission-transmission imaging. J Nucl Med. 1998;39:1996-2003.

16. Saripan MI, Petrou M, Wells K. Design of a wire-mesh collimator for gamma cameras. IEEE Trans Biomed Eng. 2007;54:1598-1612.

17. Saripan MI, Petrou M, Wells K. A comparative study on improving image resolution of the multihole collimator gamma camera modelled by the MCNPX code using the Wiener filtering technique. IEEE Eng Med Biol. 2005; $1: 34-37$.

18. Saripan MI, Hashim S, Mashohor S, Adnan WA, Marhaban MH. Characteristics of multihole collimator gamma camera simulation modeled using MCNP5. AIP. 2008;1017:205-209.

19. Ogawa K, Kato J. Wired collimator for single photon emitter. IEEE Trans Nucl Sci. 2003;50:1536-1540.

20. Briesmeister JF. MCNP Data Manual. Oak Ridge, TN: Radiation Safety Information Computational Center; 2001.

21. Jangha DN, Mintzer RA, Valentine JD. MCNP4c simulations to investigate shielding of scintillation cameras for conjugate imaging of I-123 labeled brain agents. IEEE Nucl Sci. 2001;3:1309-1312.

22. Metz CE, Atkins FB, Beck RN. The geometric transfer function component for scintillation camera collimators with straight parallel holes. Phys Med Biol. 1980;25:1059-1070.

23. Collimator Specification of ADAC Product Data. Milpitas, CA: ADAC Laboratories, Philips Medical Systems, 1999.

24. Gunter D, Matthews K, Oredonez C. The optimal design of non-parallel hole collimators. IEEE Nucl Sci. 2000;3:1344-1348.

25. De Vries DJ, Moore SC, Zimmerman RE. Development and validation of a Monte Carlo simulation of photon transport in an Anger camera. IEEE Trans Med Imaging. 1990;9:430-438.

26. Gunter DL, et al., eds. Collimator characteristics and design. In: Henkin RE, et al., eds. Nuclear Medicine. St. Louis, MO: Mosby-Year Book, Inc; 1996:96-124.

27. Li J, Jaszczak R, Turkigton TG, et al. An evaluation of lesion detectability with cone-beam, fan beam and parallel-beam collimation in SPECT by continuous ROC study. J Nucl Med. 1994;35:135-140.

28. Cot A, Sempau J, Pareto D, et al. Evaluation of the geometric, scatter and septal penetration components in fan beam collimators using Monte Carlo simulation. IEEE Nucl Sci. 2002;49:12-16. 